\title{
Research Progress of Mirna in Screening and Diagnosis of Cervical Cancer
}

\author{
Beibei Wang*, Yuejun Xu \\ Wuchang University of Technology, Wuhan, China.
}

\begin{abstract}
How to cite this paper: Beibei Wang, Yuejun Xu. (2020) Research Progress of Mirna in Screening and Diagnosis of Cervical Cancer. International Journal of Clinical and Experimental Medicine Research, 4(3), 58-62. DOI: 10.26855/ijcemr.2020.07.005
\end{abstract}

Received: May 8, 2020

Accepted: May 31, 2020

Published: June 24, 2020

*Corresponding author: Beibei Wang, Wuchang University of Technology, Wuhan, China.

Email: beibeinihaoa@hotmail.com

\begin{abstract}
In recent years, with the development of gene research, it has been found that the expression level of miRNA is related to the pathogenesis and progress of cervical cancer. MiRNA plays the role of oncogene and/or tumor suppressor gene in the occurrence and progression of cancer. The analysis of the expression spectrum and level of microRNA in cervical cancer can not only explore the correlation between microRNA and the occurrence and development of cervical cancer, but also provide new ideas for the diagnosis, treatment and prognosis of cervical cancer.
\end{abstract}

\section{Keywords}

miRNA, Cervical Cancer, Screening and Diagnosis

\section{Introduction}

Cervical cancer is a malignant tumor with high incidence in female all over the world. The incidence and mortality of cervical cancer are the fourth highest in women's cancer worldwide [1]. Besides, the incidence of cervical cancer is increasing year by year, and has a trend of youth [2]. According to the World Health Organization, there are about 490 thousand newly diagnosed cases of cervical cancer every year in the world, and about 233 thousand patients die of cervical cancer, $80 \%$ of which come from developing countries [3]. Every year, there are about 131 thousand new cases of cervical cancer in China, accounting for $28.8 \%$ of the world's new cases. The incidence and mortality of cervical cancer are younger. The incidence of women under 35 years old has increased significantly, from $5 \%$ to $10 \%$, accounting for about $1 / 3$ of new cases. The morbidity of rural women is higher than that of urban women, and the morbidity and mortality of cervical cancer in underdeveloped countries are significantly higher than those in developed countries [4]. In recent years, the incidence of cervical cancer in developed countries has been greatly reduced due to the extensive development of cervical cytology. But in some poor countries, cervical cancer is still the main cause of death. In order to explore the pathogenesis of cervical cancer, the researchers found many risk factors related to the occurrence of cervical cancer through a large number of epidemiological studies. Among these risk factors, high-risk human papillomavirus (HPV) infection is a necessary condition [5]. More than $90 \%$ of cervical cancer patients have high-risk HPV infection, but not all patients with high-risk HPV eventually develop into cervical cancer. It shows that the individual differences between HPV infected people will also affect the occurrence and development of cervical cancer, and the genetic background of the host is a very important factor [6-8]. Therefore, the occurrence and development of cervical cancer is the result of the interaction between the virus and the host [6].

The occurrence of tumor starts from the abnormal cell proliferation and apoptosis [9], which is the result of the abnormal regulation of signal pathways such as cell proliferation and apoptosis [10]. Phosphatidylase-3 (PI3K) signaling pathway is one of the most common signaling pathways with abnormal regulation in human tumors [11]. PI3K signaling pathway plays an important role in the regulation of cell cycle, glucose homeostasis, protein 
synthesis, cell proliferation and apoptosis [12]. The regulation signal of PI3K Signal Pathway originates from the phosphorylation of 3-Light group of 4,5-bisphosphonates by receptor tyrosine kinase and G-protein-coupled receptor to phosphoinositide-3,4,5-trisquamate. PIP3 can collect signal proteins (Akt, PDK1, etc.) containing PH domain (platelet leukocyte C-kinase substrate homologous domain) in the cytoplasm to the cell membrane, and promote the activation of these proteins. Activated Akt can activate downstream signal molecules through phosphorylation to regulate physiological processes such as cell proliferation and apoptosis. PTEN and SHIP can negatively regulate PI3K signaling pathway through dephosphorylation. The abnormal activation of PI3K signaling pathway is considered to be an important factor in promoting proliferation and inhibiting apoptosis of many solid tumor cells. In the study of cervical cancer cells, Akt phosphorylation level and activity were significantly increased. Yang et al. proved that overexpression of miRNA-494 in cervical cancer cells and tissues can reduce the expression of PTEN, which can inhibit the PI3K/Akt signaling pathway and lead to cell carcinogenesis [13]. As a kind of tumor suppressor gene with bispecific phosphatase activity, PTEN gene expression abnormality or mutation will cause abnormal expression of corresponding protein, thus leading to cell carcinogenesis. PTEN exists in various stages of tumor proliferation, apoptosis, migration and invasion. It negatively regulates the PI3K/Akt signaling pathway, thus inhibiting the occurrence and development of tumor [14-16]. Zhang et al. thought that PI3K/Akt signaling pathway is the downstream pathway of miRNA-218 [17]. In human colon cancer cells, miRNA-218 can inhibit PI3K and mTOR in PI3K/Akt pathway by inhibiting PI3K/Akt signaling No. 1 pathway prevents cell carcinogenesis [18-19]. PI3K is a kind of kinase that catalyzes phosphatidylinositol (PI) specifically. The activation of PI3K can cause a series of biological processes in cells, including the proliferation and differentiation of Akt and mTOR activated cells [20-21]. Zhang et al believe that miRNA-149 can regulate Akt/mTOR signaling pathway and inhibit the development of HCC [22]. The above research proves that miRNA may affect the occurrence and development of tumor by regulating the downstream signal pathway of miRNA.

\section{Biological function of miRNA}

MiRNA (microRNA, microRNA) is one of the most important noncoding regulatory molecules in eukaryotes. It is a kind of single stranded microRNA with a highly conserved gene sequence of about 18-23 nucleotides. Most miRNAs are transcribed from RNA polymerase II to PRI miRNAs with cap like structure and polyadenylated tail like structure. A few are transcribed through RNA polymerase III. The primary PRI miRNAs located in the nucleus are spliced under the action of Drosha enzyme and its auxiliary factor dgcr8, modified into pre miRNAs with about 70-100 nucleotides, which are transported to the cytoplasm by nuclear transport receptors, The PRI miRNA was cut into 22 NT size double stranded RNA by Dicer's secondary processing, which formed a nucleotide protein complex (RISC) containing mature miRNA functional chains. Mature miRNA can degrade mRNA, inhibit translation or activate transcription by binding to some complementary sequences in 3 ' UTR and 5 ' UTR of targ et mRNA [23-24]. Each miRNA may target more than 100 mRNA, while a single mRNA may contain multiple binding sites of different miRNAs [25]. MiRNA participates in and regulates many physiological processes of normal cells, such as increment, differentiation and apoptosis. It is an important factor in G1/S phase of cell cycle, which plays an important role in the growth and development, cell differentiation, material metabolism and other processes of the body. It is closely related to the occurrence and development of various diseases

\section{Serum miRNA and tumor diagnosis}

With the development of molecular genetics, molecular biology and human genomics, the research of miRNA in the mechanism of tumor occurrence and development has become a new hotspot in the field of life science. Research shows that miRNA is closely related to the occurrence and development of tumor and can be used as a potential biomarker [26]. The expression and development mechanism of miRNA in breast cancer, ovarian cancer and pancreatic cancer are relatively deep, but the research in cervical cancer is still in the exploratory stage. The analysis of miRNA expression profile and expression level in cervical cancer can not only explore the correlation between miRNA and the occurrence and development of cervical cancer, but also provide new ideas for the diagnosis, treatment and prognosis of cervical cancer. The most remarkable characteristic of miRNA is its high stability in the internal and external environment of cells and the specificity of tissue and cell source [27]. MiRNA has high conservation, timing and tissue specificity. It can express different miRNAs at a specific time, so it has better diagnostic significance for different tumors. A large number of studies have shown that miRNA can be detected in tissues, cells, serum, plasma, saliva, urine and CSF [28-30]. Liu Honglu [31] thinks that a variety of 
miRNAs in peripheral blood are early diagnostic markers of non-small cell lung cancer, esophageal squamous cell carcinoma, pancreatic cancer, head and neck squamous cell carcinoma, ovarian cancer, colorectal cancer, breast cancer, prostate cancer and blood tumor. The combined detection of multiple miRNAs in peripheral blood and the combined detection of tumor specific miRNAs, serology, imaging and other auxiliary means can improve the sensitivity and specificity of diagnosis for tumor in early stage. The number of miRNA is relatively small and the sensitivity of small molecule detection is higher than that of traditional protein markers. Therefore, miRNA has prominent advantages in tumor diagnosis, and it is suitable for the establishment of rapid and convenient clinical detection methods. Serum microRNAs have wide application prospects in the development of disease diagnosis methods [32]. Most RNA is easily degraded by RNA enzyme, but the sequence of serum miRNA is short and stable, and it is difficult to degrade after long-term preservation and boiling with strong acid and alkali resistance $[8,14,15]$, and has high sensitivity [27, 33, 34]. Therefore, serum miRNA can be used as a tumor marker for early screening, early diagnosis and prognosis of cervical cancer.

\section{Diagnostic value of serum miRNA in cervical cancer}

At present, the clinical diagnosis of cervical cancer mainly depends on the "three steps" procedure, that is, cervical cytology and/or HPV detection, colposcopy, cervical biopsy. The clinical diagnosis of cervical cancer is expensive, time-consuming, traumatic and the patients' compliance is poor. However, the detection of serum miRNA is convenient, rapid and repeatable, which is beneficial to the clinical diagnosis of cervical cancer. In recent years, many scholars at home and abroad began to study the expression of miRNA in cervical cancer and its mechanism of occurrence and development. Renfei et al. [35] showed that miRNA-574-5p was highly expressed in the serum of patients with cervical cancer. The higher the pathological grade, the larger the tumor diameter, and it had a regulatory effect on the progression of cervical cancer. Li et al. [36] proved for the first time that serum miRNA-486-5p was significantly over expressed in the serum and tissues of cervical cancer patients. It could inhibit PTEN expression and activating the PI3K/Akt pathway, stimulate cell proliferation, migration and invasion. Therefore, serum miRNA-486-5p is a new molecular biomarker and has potential in the diagnosis of cervical cancer. Similar studies have found that miRNA-21 is up-regulated in cervical cancer tissues and serum, and promotes the proliferation, survival, migration and invasion of cervical cancer cells by targeting TIMP3 [37].

High risk human papillomavirus (HPV) persistent infection is a clear pathogenic factor of cervical cancer. It can change the expression of miRNAs by oncogenes or tumor suppressor genes, and it can be a target for early diagnosis of cervical cancer. In recent years, more and more scholars have studied the changes of miRNA expression in cervical cancer patients with HPV infection. According to Chang Chunhong's study, the abnormal high expression of plasma miRNA-155 in HPV positive cervical cancer patients can be used as a biological marker for early diagnosis and differential diagnosis of CIN [38]. Feng Lei et al. found that the level of plasma mirna-10b and SCC antigen increased, which is expected to be a molecular marker for early diagnosis of cervical cancer. The combination of miRNA-10b and SCC antigen and CEA can improve the accuracy of diagnosis of cervical cancer [39]. Therefore, serum miRNA has certain value in the diagnosis of HPV positive cervical cancer.

\section{Serum miRNA and prognosis of cervical cancer}

The main factor of poor prognosis of cervical cancer is lymph node metastasis, which mainly detected by MRI, CT and other imaging. However, the detection of small lesions has certain limitations. At present, there is no specific marker to guide the prognosis of cervical cancer. The research shows that miRNA is closely related to the prognosis of cervical cancer. MiRNA is conducive to the prognosis evaluation of cervical cancer. Gu et al. suggest that the expression of miRNA-205 in serum is up-regulated in cervical cancer, which is a biomarker to predict the prognosis of cervical cancer patients [40]. Six kinds of serum microRNAs (miR-1246, miR-20a, miR2392, miR-3147, miR-3162-5p and miR-4484) which can predict the lymph node metastasis of cervical cancer patients were identified. Their predictive value is lower than that of cervical cancer tissues, but higher than that of squamous cell carcinoma antigen analysis. It is considered that serum miRNA is a predictive index of early cervical cancer with lymph node metastasis.

\section{Conclusion}

In conclusion, the abnormal expression of miRNA in cervical cancer is closely related to the occurrence, development and prognosis of cervical cancer. At present, the research cost of detecting miRNA is high and samples are limited. It is still necessary to select appropriate tumor markers for early diagnosis and prognosis of 
cervical cancer, and the regulatory mechanism of miRNA in cervical cancer is still unclear, which needs further exploration and research.

\section{References}

[1] Bray F, Ferlay J, Soerjomataram I, et al. (2018). Global cancer statistics 2018: GLOBOCAN estimates of incidence and mortality worldwide for 36 cancers in 185 countries [J]. CA Cancer J Clin, 68(6): 394-424.

[2] Xie Shanyan and Ren Peng. (2018). Trend analysis and corresponding countermeasures of cervical cancer [J]. Journal of Raditional Chinese Medicine Management, 26(5): 10-12.

[3] Crow J M. (2012). HPV: the global burden: human papillomavirus (HPV) has become synonymous with cervical cancer, but its actual footprint is much bigger [J]. Nature, 488(7413): S2-S2.

[4] Reshmi G, Pillai M R. (2008). Beyond HPV: oncomirs as new players in cervical cancer [J]. FEBS Letters, 582(30): 4113-4116.

[5] Kobayashi T. (2003). [Obstetrics and gynecology] [J]. 61(10): 1787-92.

[6] De Freitas A C, Gurgel A P A D, Chagas B S, et al. (2012). Susceptibility to cervical cancer: an overview [J]. Gynecologic Oncology, 126(2): 304-311.

[7] Fang J, Li Y, Zhang J, et al. (2017). Correlation between polymorphisms in microRNA-regulated genes and cervical cancer susceptibility in a Xinjiang Uygur population [J]. Oncotarget, 8(19): 31758.

[8] Zeng X, Zhang Y, Yue T, et al. (2017). Association between XRCC1 polymorphisms and the risk of cervical cancer: a meta-analysis based on 4895 subjects [J]. Oncotarget, 8(2): 2249.

[9] Thompson C B. (1995). Apoptosis in the pathogenesis and treatment of disease [J]. Science, 267(5203): 1456-1462.

[10] Manzo-Merino J, Contreras-Paredes A, (2014). Vázquez-Ulloa E, et al. The role of signaling pathways in cervical cancer and molecular therapeutic targets [J]. Archives of Medical Research, 45(7): 525-539.

[11] Martini M, De Santis M C, Braccini L, et al. (2014). PI3K/AKT signaling pathway and cancer: an updated review [J]. Annals of Medicine, 46(6): 372-383.

[12] Katso R, Okkenhaug K, Ahmadi K, et al. (2001). Cellular function of phosphoinositide 3-kinases: implications for development, immunity, homeostasis, and cancer [J]. Annual Review of Cell and Developmental Biology, 17(1): 615-675.

[13] Yang Y K, Xi W Y, Xi R X, et al. (2015). MicroRNA-494 promotes cervical cancer proliferation through the regulation of PTEN[J]. Oncology Reports, 33(5): 2393-2401.

[14] Zhang S, Yu D. (2010). PI (3) king apart PTEN’s role in cancer [J]. Clinical Cancer Research, 16(17): 4325-4330.

[15] Stewart A L, Mhashilkar A M, Yang X H, et al. (2002). PI3K blockade by Ad-PTEN inhibits invasion and induces apoptosis in radial growth phase and metastatic melanoma cells [J]. Molecular Medicine, 8(8): 451-461.

[16] Tamura M, Gu J, Matsumoto K, et al. (1998). Inhibition of cell migration, spreading, and focal adhesions by tumor suppressor PTEN [J]. Science, 280(5369): 1614-1617.

[17] Zhang X, Shi H, Tang H, et al. (2015). miR-218 inhibits the invasion and migration of colon cancer cells by targeting the PI3K/Akt/mTOR signaling pathway [J]. International Journal of Molecular Medicine, 35(5): 1301-1308.

[18] Hales E C, Taub J W, Matherly L H. (2014). New insights into Notch1 regulation of the PI3K - AKT - mTOR1 signaling axis: Targeted therapy of $\gamma$-secretase inhibitor resistant T-cell acute lymphoblastic leukemia[J]. Cellular Signalling, 26(1): 149-161.

[19] Kim S M, Park J H, Kim K D, et al. (2014). Brassinin induces apoptosis in PC - 3 human prostate cancer cells through the suppression of PI3K/Akt/mTOR/S6K1 signaling cascades [J]. Phytotherapy Research, 28(3): 423-431.

[20] Jung K H, Yan H H, Fang Z, et al. (2014). HS-104, a PI3K inhibitor, enhances the anticancer efficacy of gemcitabine in pancreatic cancer [J]. International journal of oncology, 45(1): 311-321.

[21] Slotkin E K, Patwardhan P P, Vasudeva S D, et al. (2015). MLN0128, an ATP-competitive mTOR kinase inhibitor with potent in vitro and in vivo antitumor activity, as potential therapy for bone and soft-tissue sarcoma[J]. Molecular cancer therapeutics, 14(2): 395-406.

[22] Zhang Y, Guo X, Xiong L, et al. (2014). Comprehensive analysis of microRNA-regulated protein interaction network reveals the tumor suppressive role of microRNA-149 in human hepatocellular carcinoma via targeting AKT-mTOR pathway [J]. Molecular Cancer, 13(1): 253.

[23] Tsu V, Jeronimo J. (2016). Saving the World’s Women from Cervical Cancer [J]. N Engl J Med, 374(26): $2509-2511$.

[24] Gommans W M, Berezikov E. (2012). Controlling miRNA regulation in disease [J]. Methods Mol Biol, 822: 1-18.

[25] Podshivalova K, Salomon D R. (2013). MicroRNA regulation of T-lymphocyte immunity: modulation of molecular networks responsible for T-cell activation differentiation and development [J]. Crit Rev Immunol, 33(5): 435-476.

[26] Fries GR, Zhang W, Benevenuto D, et al. (2019). MicroRNAs in Major Depressive Disorder [J]. Adv Exp Med Biol, 1118: 
175-190.

[27] Sathyanarayanan A, Chandrasekaran K S, Karunagaran D. (2016). microRNA-146a inhibits proliferation migration and invasion of human cervical and colorectal cancer cells [J]. Biochem Biophys Res Commun, 480(4): 528-533.

[28] Reddy K B. (2015). MicroRNA (miRNA) in cancer [J]. Cancer Cell Int, 15: 38.

[29] Abba M L, Patil N, Leupold J H, et al. (2017). MicroRNAs as novel targets and tools in cancer therapy [J]. Cancer Lett, 387: 84-94.

[30] Arroyo J D, Chevillet J R, Kroh EM, et al. (2011). Argonaute2 complexes carry a population of circulating microRNAs independent of vesicles in human plasma [J]. Proc Natl Acad Sci USA, 108(12): 5003-5008.

[31] Ma Y, Liang AJ, Fan YP, et al. (2016). Dysregulation and functional roles of miR-183-96-182 cluster in cancer cell proliferation invasion and metastasis [J]. Oncotarget, 7(27): 42805-42825.

[32] Zhang W, Qian P, Zhang X, et al. (2015). Autocrine Paracrine Human Growth Hormone-stimulatedMicroRNA96-182-183 Cluster Promotes Epithelial-Mesenchymal Transition and Invasion in Breast Cancer [J]. J Biol Chem, 290(22): 13812-13829.

[33] Wang Fan, Feng Yuzhen, Zhao Huan, Zhou Mengxia. (2013). Advances in Research of miR-183 Family and Cancer. J Int Obstet Gynecol, 40(5): 436-439.

[34] Qiu Liwei, Wang Li, pan Liuyi, Yang Xuli, Gu Juanjuan, Yang Junling, Yao Dengfu. (2015). Expression of miRNA-183 family in circulating blood of patients with liver cancer and its clinical value. China Cancer Society, China Cancer Society hepatoma Committee, cancer group of oncology branch of Chinese Medical Association: China Cancer Society, 2015: 2.

[35] Ren Fei, Yao Desheng, Chen Junying. (2015). Serum mirna-574-5p level and its diagnostic value in patients with cervical squamous cell carcinoma [J]. GUANGXI MEDICAL JOURNAL, 37(01): 15-17.

[36] Rischin D, Narayan K, Oza AM, et al. (2010). Phase 1 study of tirapazamine in combination with radiation and weekly cisplatin in patients with locally advanced cervical cancer [J]. Int J Gynecol Cancer, 20(5): 827-833.

[37] Bouvard V, Baan R, Straif K, et al. (2009). A review of human carcinogens-Part B: biological agents [J]. Lancet Oncol, 10(4): 321-322.

[38] Chang Chunhong, Feng Lei, Guan Xiaoqing, et al. (2018). Expression and clinical significance of miR-155 in human papillomavirus positive cervical cancer and cervical intraepithelial neoplasia [J]. Journal of PLA Medical College, 39(03): $243-247$.

[39] Feng Lei, Chang Chunhong, Guan Xiaoqing. (2018). Expression and clinical significance of mirna-10b in patients with cervical cancer [J]. Journal of Modern Laboratory Medicine, 33(01): 52-55.

[40] Ma Q, Wan G, Wang S, et al. (2014). Serum microRNA-205 as a novel biomarker for cervical cancer patients [J]. Cancer Cell Int, 14: 81. 